\title{
Numerical Simulation and Optimization of Hole Spacing for Cement Grouting in Rocks
}

\author{
Ping Fu, Jinjie Zhang, Zhanqing Xing, and Xiaodong Yang \\ China Institute of Water Resources and Hydropower Research, Beijing 100038, China \\ Correspondence should be addressed to Ping Fu; fuping@iwhr.com
}

Received 30 July 2013; Accepted 25 August 2013

Academic Editor: Ga Zhang

Copyright ( 2013 Ping Fu et al. This is an open access article distributed under the Creative Commons Attribution License, which permits unrestricted use, distribution, and reproduction in any medium, provided the original work is properly cited.

\begin{abstract}
The fine fissures of V-diabase were the main stratigraphic that affected the effectiveness of foundation grout curtain in Dagang Mountain Hydropower Station. Thus, specialized in situ grouting tests were conducted to determine reasonable hole spacing and other parameters. Considering time variation of the rheological parameters of grout, variation of grouting pressure gradient, and evolution law of the fracture opening, numerical simulations were performed on the diffusion process of cement grouting in the fissures of the rock mass. The distribution of permeability after grouting was obtained on the basis of analysis results, and the grouting hole spacing was discussed based on the reliability analysis. A probability of optimization along with a finer optimization precision as $0.1 \mathrm{~m}$ could be adopted when compared with the accuracy of $0.5 \mathrm{~m}$ that is commonly used. The results could provide a useful reference for choosing reasonable grouting hole spacing in similar projects.
\end{abstract}

\section{Introduction}

The Dagang Mountain Hydropower Station, located in Shimian county of Yaan City, is the 14th cascade hydropower station in the main stream of the Dadu River in Sichuan province. grout curtain is more than 450,000 meters in length, and the main rock for grouting is granite. Parts of the grout curtain passed through complex geological conditions, containing V-diabase dikes, slightly fractured and contacted with granite in fault type. This may have a significant influence on the local grout curtain. Therefore, in situ curtain grouting tests were conducted to determine the reasonable hole spacing and other parameters in the curtain grouting.

The grout diffuses from the grouting hole to the rock cracks under grouting pressure in the batholith. The distance between the neighboring grouting holes is determined by the diffusion distance of the grout, which is also the most important reference for selecting the other technical parameters and for evaluating the effect of the grouting. Due to the complex and volatile characteristics of rock mass and the hidden diffusion grouting process, it is difficult to monitor the diffusion process during the construction. Moreover, the previous theoretical and empirical formulas for calculating the diffusion distance of grout were far from mature. Thus, the hole spacing is usually selected on the basis of engineering experiences among several limited numbers with the accuracy of $0.5 \mathrm{~m}$, namely, $1.5 \mathrm{~m}, 2 \mathrm{~m}, 2.5 \mathrm{~m}$, and $3 \mathrm{~m}$. With the development of computation techniques, the numerical simulation methods were used to calculate the grouting process with the focus on the grouting technology [1-6]. The flow formula of Bingham slurry in onedimensional horizontal fissure has been conducted, and the stiff plug in the centre of the flow was considered as one of the grouting stop criteria by Xiaodong in 1987 [7]. The diffusion equation based on the continuity equation and equilibrium equation has been conducted in recent years [814]. Serialized studies have been conducted on the grouting using the numerical simulation. However, these researches placed their emphasis on the calculation of the diffusion radius for single grouting fissure. In other words, they failed to consider the time variability of rheological parameters of cement grout, the fracture opening changes, and the precipitation law and the consolidation law of cement grout. There was not quantitative analysis to evaluate the effect of the grouting in the multifractured rock mass. Therefore, it is difficult to establish the macroscopical relationship between 
the grout diffusion radius (grouting hole spacing) and the effect of the grouting. The analysis results were still far away from the engineering requirements.

In this paper, a fracture network of rock mass was established for dual medium grouting by Monte Carlo random method. The finite element analysis was conducted for the diffusion process in the fissure and the permeability of rock mass after grouting. The grouting hole was accordingly optimized and evaluated on the basis of analysis results. The analysis was performed based on the diffusion motion equation of cement grout in a single fracture, with full consideration of various factors such as the time variability of rheological parameters of cement grout, the opening change and the fissure cracking in the grouting process, and the precipitation of cement particles.

\section{Diffusion Equations of Cement Grout in the Single Fracture}

2.1. Diffusion Equation. Cement grout in rock mass is essentially a two-phase flow process of the granular liquid in the fissures. According to the flow conservation and balance equation, the diffusion motion equation of grout in the single fissure can be deduced as follows [12-14]:

$$
u=-\frac{J b^{2}}{2 \eta}\left[1-\left(\frac{z}{b}\right)^{2}-2 \frac{z_{b}}{b}\left(1-\frac{z}{b}\right)\right],
$$

where $u$ is the velocity of the grout movement at a certain cross-section point; $z$ is the distance of a certain point from the center on the cross-section; $z_{b}$ is a half of the height of the plug flow; $b$ is a half of the fracture opening on a cross-section; $\eta$ is the viscosity coefficient of the grout; $J$ is the pressure gradient.

To integrate the above equation along the fractured crosssection, the grout flow in a single fracture at a certain moment can be obtained as follows:

$$
q_{i}=\frac{2 J b^{3}}{3 \eta}\left[1-\frac{3 z_{b}}{2 b}+\frac{1}{2}\left(\frac{z_{b}}{b}\right)^{3}\right] .
$$

Obviously, by adding up all the fracture flow $q_{i}$, the injection rate of grouting at a certain moment, $U$, is obtained, namely,

$$
U=\sum_{i}^{t} q_{i}
$$

To make a time integration of the injection rate, grout quantity, $Q$, can be obtained, namely,

$$
Q=\int_{0}^{t} \sum_{i}^{t} q_{i} d t
$$

When $t$ is the end time of grouting, $Q$ is the total injection amount of grout.

2.2. Evolution Rules of the Grouting Parameter. From the diffusion equation of a single fissure, it can be seen that the flow $q$ is dependent on the fracture opening, the pressure gradient, and the rheological parameters of grout. All these factors are always changing throughout the grouting process.

2.2.1. Time Variation of the Rheological Parameters of Grout. The rheological parameters of cement grout are time varying and depend significantly on the grouting time, the water-cement ratio, and the water temperature. The pure cement grout within the range of the commonly used watercement ratio is a typical Bingham rheological material, whose essential features are having structural strength and timedependent performance [15-18]. To describe the grout rheological model approximately, the following linear equation can be used:

$$
\tau=\tau_{0}(t)+\eta(t) \dot{\gamma}
$$

where $\tau$ and $\dot{\gamma}$ are the shear stress and the strain rate of grout, respectively; $\tau_{0}(t)$ and $\eta(t)$ are the time-dependent yielding strength (dynamic shear force) and the plastic viscosity of grout, respectively.

Different water-cement ratios were used in the grouting tests of Dagang Mountain Hydropower Station. The rheological parameters and time curve were measured by the long homemade capillary rheological parameter meter (Figure 1).

2.2.2. Variation of Pressure Gradient. The rock fracture for grouting is usually filled with groundwater. It was assumed that there was not exchange between the grouting front and the groundwater, and thus there was only hydrostatic pressure. The grouting pressure, in addition to be affected by the local head loss and frictional head loss, will push grout flow in the fracture. Its gradient in the fracture is directly dependent on the attenuation of grouting pressure and the diffusion radius. The variation of pressure gradient could be investigated in the numerical simulation of grout diffusion in the fracture.

2.2.3. Evolution law of the Fracture Opening. The fracture opening is the principal variation in the diffusion equation, which plays a leading role in the diffusion distance [19]. The evolution law of the following five factors in the grouting process directly affects the accuracy of the numerical simulation for the diffusion distance.

(1) Effect of the Fracture Roughness. Seepage channels of the grout are mainly the fracture surfaces that are rough and often contain parts of the cementation or filling. This leads to complicated fracture opening with a large influence on the fluid motion. In the fracture hydraulics, the mechanical opening of the fracture, $2 b_{m}$, is replaced by the equivalent hydraulic opening, $2 b_{h}$, which is defined as follows: under the same pressure gradient and flow pattern, the volume flow within the rough fracture is equal to that within the flat and smooth fracture whose opening is $2 b_{h} .2 b_{h}$ can be determined comprehensively by the drilling hydraulic document and water pressure test. As a virtual opening, the equivalent hydraulic opening reflects the hydraulic characteristics of 


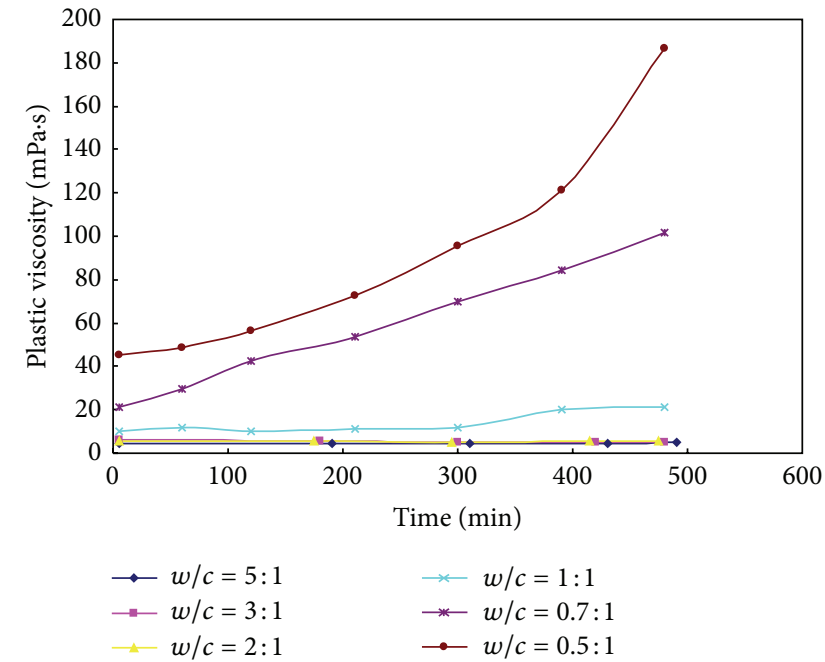

(a) Plastic viscosity of grout

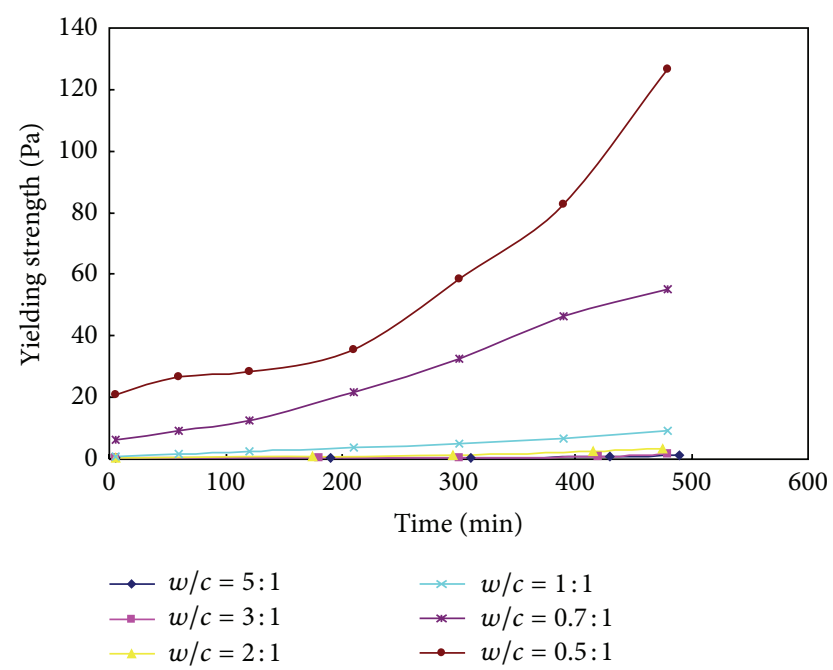

(b) Yielding strength of grout

FIGURE 1: Time variation curves of the rheological parameters for cement grouts at different ratios $(w / c$ is the water-cement ratio of grout).

fractures and shares some internal relations with the mechanical opening $2 b_{m}$. Renshaw (1995) deduced the following equation based on the probability and statistics theory [20]:

$$
\frac{b_{h}}{b_{m}}=\left[\left(\frac{\sigma_{b}}{b_{m}}\right)^{2}+1\right]^{-1 / 2}=\left[\frac{\exp \sigma_{B}^{2} \cdot\left(\exp \sigma_{B}^{2}-1\right)}{\left[\exp \left(\sigma_{B}^{2} / 2\right)\right]^{2}}+1\right]^{-1 / 2},
$$

where $\sigma_{b}$ and $\sigma_{B}$ are the standard deviation of the opening and the standard deviation of the opening to the values, respectively.

(2) Change of the Fracture Opening due to the Precipitation of Cement Particles. The analysis of the instable grout in fractured rock showed that the fracture filled with grout material is mainly caused by the cement particle precipitation. There is a critical velocity value, $V_{\mathrm{kp}}$, in the flow process of cement grout. When the velocity of grout diffusion is less than $V_{\mathrm{kp}}$, the cement particles begin to precipitate, the sediment at the bottom of the fractured wall gradually increases, and the effective opening of the fracture gradually decreases. The reduction of the fracture opening will further slow the grout flow down. The particles will continue to precipitate until the fracture opening becomes less than $0.2 \mathrm{~mm}$, when the cement particles cannot pass through the fractures, and thus the grout seepage channel is regarded to be blocked. The semiempirical formula for the critical velocity value is [21]

$$
V_{\mathrm{kp}}=k(g \delta)^{0.5}\left[\frac{V^{2}\left(\rho_{T}-\rho_{B}\right) \sigma^{m}}{6 f g d_{\mathrm{cp}} \rho_{B}}\right]^{3 / 7},
$$

where $k$ is the correction coefficient; $g$ is the gravitational acceleration; $V$ is the sinking velocity of cement particles in water; $\delta$ is the fracture opening; $\rho_{T}$ and $\rho_{B}$ are the density of the cement particles and water, respectively; $\sigma$ is the content of solid particles in solution; $f$ is the resistance coefficient

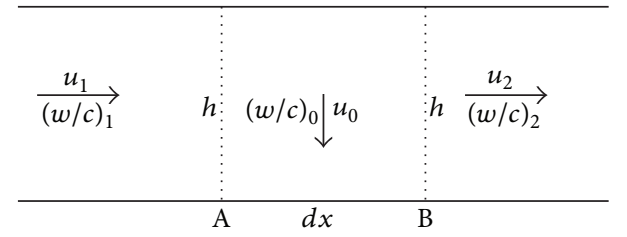

FIGURE 2: Change of the water-cement ratio after the cement particles precipitation.

of water in the fracture; $d_{c p}$ is the feature size of the cement particle; $m$ is the empirical indicators.

The sinking velocity of cement particles in water $V$ can be approximately regarded as obeying the Stokes' law for free settling [14]:

$$
V=\frac{d^{2}\left(\rho_{T}-\rho_{B}\right) g}{18 \eta},
$$

where $d$ is the mean size of the cement particles and $\eta$ is the viscosity of the liquid medium or grout.

To understand the cement particles precipitation phenomenon from another angle, it is also a process of the increase of the water-cement ratio caused by free water separating out of grout, leading to the change of rheological parameters of grout. The flow and precipitation process was shown in Figure 2.

According to the conservation of cement mass, the following formula can be obtained:

$$
\left(\frac{w}{c}\right)_{0}=h \cdot \frac{0.316+(w / c)_{1}}{u_{0} t+h-1.5 W_{1}}-0.316
$$

where $(w / c)_{1}$ is the initial water-cement ratio of the grout; $(w / c)_{0}$ is the water-cement ratio of the grout after precipitation; $t$ is the settling time started from the precipitation; $u_{0}$ 
is the velocity of cement particle precipitation in the grout; $h$ is the fracture opening. The physical meaning of $W_{1}$ is the height accumulated from the precipitation of cement particles in the grout and is described as follows:

$$
W_{1}=\frac{u_{0} t}{0.1+0.316 \cdot(w / c)_{1}}
$$

obviously, given that

$$
h-1.5 W_{1}<0.2 \mathrm{~mm} \text {. }
$$

The fracture has been blocked by the cement particles if (11) is satisfied; this demonstrates that the fracture cannot be grouted.

(3) Change of the Fracture Opening Caused by the Rock Mass Deformation. In the process of grout diffusion, the grouting pressure always exerts on the upper and lower surfaces of the fissure, tending to make the fissures open. Meanwhile the fissure is constrained by the surrounding rock mass. The displacements of the upper and lower surfaces of the fissure at a particular moment can be calculated through the finite element model that was set up in the entire grout diffusion area by the composite element method containing the fissure network [14].

(4) Opening Change Led by the Fissure Cracking and Expansion. In the process of grout diffusion, when the grouting pressure reaches a critical value due to the stress concentration at the crack tip, the cracking will emerge, resulting in the increase of fracture opening. The grouting pressure is always applied to the fractured surfaces in the form of the surface force, and its direction is normal to the surface. The grouting pressure, serving as the inner water pressure, exerts approximate symmetrical distribution of the force on the upper and lower surfaces of the crack, increasing the fracture opening through tension. On the other hand, shear extension happened to the fracture surface due to the grouting pressure. Therefore, for the ordinary characteristics of stratum, the failure of rock mass is always shear failure rather than tension failure under the grouting pressure. The fracture extends along the original fracture direction, which is type I cracking with cracking angle of zero. When the stress intensity factor $K_{\mathrm{I}}$ reaches a critical value $K_{\mathrm{Ic}}$, the crack will extend unsteadily [22], which is the cracking criterion at the crack tip in the grouting process. When calculating the fracture unit in the finite element analysis, the stress intensity factor, $K_{\mathrm{I}}$, can be obtained using the following formula:

$$
K_{\mathrm{I}}=\lim _{r \rightarrow 0} \frac{G(1+\mu)}{(1-\mu)} \sqrt{\frac{2 \pi}{r}} u,
$$

where $G$ is deformation modulus of rock mass; $\mu$ is Poisson's ratio; $r$ is the distance between the fissure and the cracking point; $u$ is the displacements of fissures. The fracture toughness, $K_{\mathrm{Ic}}$, is the inherent characteristics of material and is usually determined by using the experimental methods. Its value is

$$
K_{\text {Ic }}=\sqrt{\frac{2 E W_{r}}{1-\mu^{2}}},
$$

where $E$ is the elastic modulus of rock mass and $W_{r}$ is the rock-specific surface energy.

(5) Fracturing Opening of the Same Fissure at Different Locations. There are some differences in the spatial distribution of the fissures; namely, the fracture openings are different at different locations. The fracture opening changes when the grout diffuses.

\section{Simulation Model}

Fissures with various geometric characteristics are often contained in the rock mass, and the diffusion distances are also different in different fissures. Therefore, the diffusion and filling of grout in different fractures within a certain distance from the drilling hole should be calculated, and its grouting effect should be analyzed. To analyze the entire grouting effect for the rock mass, the fracture network and computing medium model that can reflect the characteristics of grouting were established in this paper.

3.1. Simulation of the 3D Fracture Network. According to the geometric characteristics of rock mass, the three-dimensional fracture network should be consistent with the facture network of site as much as possible. Monte-Carlo method has been widely used to generate the 3D fracture network [23]. Compared with other engineering applications, grouting works can simply simulate the fracture network near grouting section. The geometric parameters of fracture comply with a certain distribution function whose simulation is to randomly generate a sample. However, concerning the fact that significant differences may exist between the generated network and the practical situation, it is necessary to amend the fracture opening with the greatest impact.

3.1.1. Extreme Value Test. Limiting the simulated fissures of large openings based on the geological survey data or downhole television data is essential so as to prevent the unreasonable ones from generating.

3.1.2. Acoustic Wave Test. The acoustic wave test refers to the calculation of average acoustic velocity according to the distance between two points of rock mass and the time for acoustic wave propagation. The rock mass between two points consists of the rock framework (matrix) and the pore filled with air or liquid. Both the distance between two points and the acoustic wave velocity in rock mass, fresh rock, and water or air are known. Therefore, the sum of the fissure openings can be calculated by using the following formula:

$$
\sum l_{2}=\frac{\left(v_{1}-v\right) \cdot v_{2}}{\left(v_{1}-v_{2}\right) \cdot v} \cdot l
$$

where $\sum l_{2}$ is the sum of the fissure openings; $l$ is the distance between two points of rock mass; $v$ is the acoustic wave velocity in rock mass; $v_{1}$ is the acoustic wave velocity in fresh rock; $v_{2}$ is the acoustic wave velocity in water or air. 
Compared with the sum of fissure openings obtained from the numerical simulation, it can be analyzed whether the sum of fissure openings in fracture network is suitable.

3.1.3. Water Pressure Test. A simple water pressure test is made usually before grouting. The flow equation of water in a single fracture meets the cubic law [24] as follows:

$$
q=\frac{2 J b^{3}}{3 \eta}
$$

where $q$ is the water flow in a single fracture; $b$ is half of the fracture opening on a cross-section; $\eta$ is the viscosity coefficient of water; $J$ is the water test pressure gradient.

To sum up the simulated crack flow, the injection rate at a certain moment can be obtained (3). When $t=20 \mathrm{~min}$ after the loop calculation, $U$ is the stable flow which meets the law of simple water pressure test, and the permeability rate of the fracture network in simulation can be calculated by the Lugeon calculation formula. Comparing this permeability rate with the results of water pressure test, it can be examined whether the distribution law of fracture opening in the fracture network is consistent with the practical situation.

3.2. Dual Medium Model of Grouting. As for the practice and theory in grouting engineering, it is believed that the pores whose sizes are less than three times $D_{85}$ of material cannot be strictly grouted. In other words, the cracks whose openings are less than $0.2 \mathrm{~mm}$ cannot be strictly grouted using the ordinary Portland cement-based materials. The Distribution of fracture opening mostly follows the negative exponential distribution [23], and the microfine fissures less than $0.2 \mathrm{~mm}$ account for a higher proportion. In the grouting process, the cement particles are difficult to enter these microfine fissures. Serving as channels for draining excessive water in the grouting process, the microfine fissure are always in a state of compression under the grouting pressure. At the same time, based on the law of Water Cube, the grout diffusion is mainly controlled by the large opening fissures. In order to reduce the amount of calculation, those fissures with the openings greater than $0.2 \mathrm{~mm}$ were considered as the structural planes and thus have the strength and the deformation characteristics of structural planes. Those fissures with the openings less than $0.2 \mathrm{~mm}$ are believed to distribute uniformly in rock mass. Thus, a dual medium model of grouting reflecting the characteristics of grouting was established if the strength parameters and the deformation parameters of the microfine fissures are distributed to the surrounding rock mass equivalently.

3.3. Evaluation Indicator of the Grouting Effect. The permeability rate after grouting serves as the main indicator of grout curtain evaluation. The rock mass after grouting can still be divided into two parts: the grouted rock and the nongrouted fissure. The grouted rock is regarded as homogeneous continuous medium with a weak water permeability. The nongrouted fissures and the microfine fissures with the opening less than $0.2 \mathrm{~mm}$ have strong flow capacity, play the main role of hydraulic conductivity, and serve as important seepage channels. The simulation method for the water pressure test (15) can be used to investigate the water permeability rate at different locations of rock mass after grouting.

\section{Simulation of the V-Diabase in Dagang Mountain Hydropower Station}

According to the basic model of grouting simulation, combined with the program of the finite element method, the grouting process was simulated with analysis of its effect. The simulation took advantages of the relevant grouting test data of the V-diabase in the Dagang Mountain Hydropower Station.

4.1. Basic Conditions. The 1-1 hole in the downstream of original grouting within the grouting-test area was selected for the simulation of the permeability coefficient of rock mass after grouting. The grouting depth is $55.6 \mathrm{~m}$. However, the simulated grouting depth ranged from $5.5 \mathrm{~m}$ to $55.6 \mathrm{~m}$ in order to reduce the influence of the abnormal grouting such as the oozing grout and the colluding grout. The integrity of the granite in this area with the depth from $0 \mathrm{~m}$ to $60 \mathrm{~m}$, which mainly consisted of a steep fissure group and a gentle sloping fissure group, is poor. The maximum opening of the fissures is $0.5 \mathrm{~cm}$; the acoustic velocity of fresh rock is $6500 \mathrm{~m} / \mathrm{s}$; the average velocity of grouting stage, the water permeability before grouting, and other parameters are shown in Table 1 . The design grouting hole spacing is $2 \mathrm{~m}$, and the design impermeable standard is less than $1 \mathrm{Lu}$.

4.2. Computed Results. The simulations of the grouting process for ten grouting stages were conducted. The computed results of the grouting process in Stage 9 are shown in Figure 3. A comparison between the simulation results and the results of practical grouting process during the grouting is shown in Table 2. A comparison of the grouting effect between the simulation results and the measurement results of practical grouting process after grouting is shown in Figure 4.

The results in Table 2 and Figure 4 showed that relative error or absolute error between the numerical simulation and the measurement results was acceptable, including the injection rate, the cumulative amount of grouting, and the accumulated injecting cement content. This indicated that the simulated grouting process basically reflected the practical grouting process. In addition, through comparing the simulated results of the water permeability and the sound wave velocity after grouting with that of the practical grouting, the treatment effect of cement grouting on the V-diabase rock mass could be reflected fundamentally using the numerical simulation.

The single hole calculation results were used to evaluate the effect of the grouting. The permeability rate at a certain distance from the drilling center was analyzed for a quantitative evaluation on the grouting's effect. The corresponding distances of $1 \mathrm{Lu}$ and $3 \mathrm{Lu}$ in different grouting stages were 


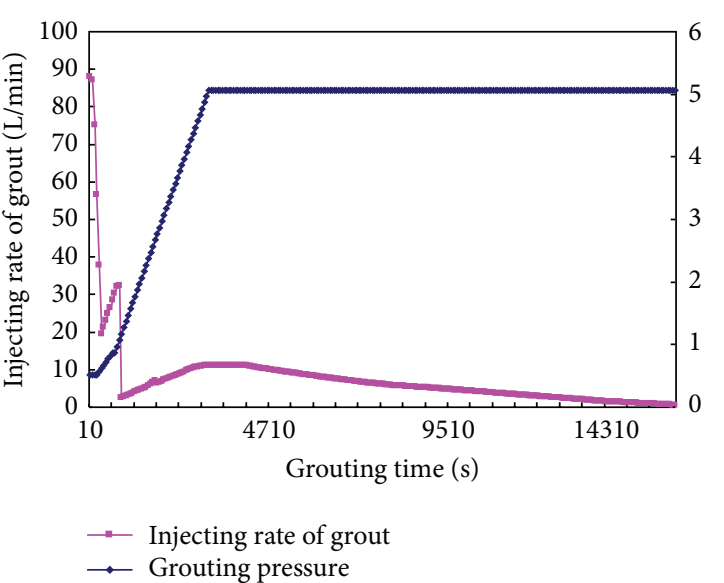

(a)

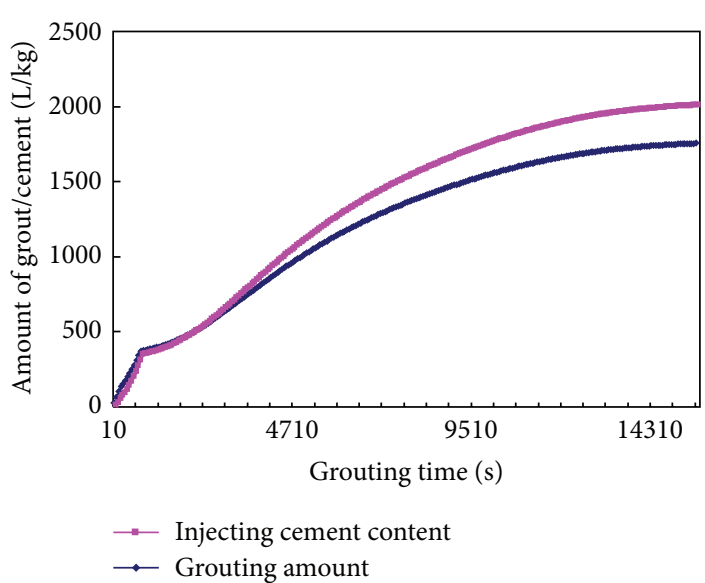

(b)

FIGURE 3: Computed results of grouting in Stage $9(45.5-50.5 \mathrm{~m})$.

TABLE 1: Acoustic wave velocity and the permeability rate of drilling holes before grouting.

\begin{tabular}{lcccccccccc}
\hline Grout stage & Stage 1 & Stage 2 & Stage 3 & Stage 4 & Stage 5 & Stage 6 & Stage 7 & Stage 8 & Stage 9 & Stage 10 \\
\hline Drilling hole/m & $5.5-10.5$ & -15.5 & -20.5 & -25.5 & -30.5 & -35.5 & -40.5 & -45.5 & -50.5 & -55.6 \\
Wave velocity/m/s & 5140 & 5119 & 4629 & 4565 & 5077 & 4994 & 5061 & 5053 & 4816 & 4756 \\
Permeability rate/Lu & 9.94 & 11.61 & 10.66 & 8.33 & 11.15 & 8.36 & 7.17 & 9.82 & 9.2 & 5.56 \\
\hline
\end{tabular}

TABLE 2: Simulation results in each grouting stage.

\begin{tabular}{lccccccccccc}
\hline \multicolumn{2}{c}{ Grouting stage } & Stage 1 & Stage 2 & Stage 3 & Stage 4 & Stage 5 & Stage 6 & Stage 7 & Stage 8 & Stage 9 & Stage 10 \\
\hline \multirow{3}{*}{ Pressure/Pa } & Simulation & 2.46 & 2.83 & 3.08 & 3.38 & 3.99 & 4.67 & 5.02 & 5.13 & 5.07 & 4.23 \\
& Measurement & 2.51 & 2.88 & 3.13 & 3.43 & 4.04 & 4.72 & 5.07 & 5.18 & 5.07 & 5.08 \\
& Difference \% & -2.0 & -1.7 & -1.6 & -1.5 & -1.2 & -1.1 & -1.0 & -1.0 & 0.0 & -16.7 \\
\hline \multirow{3}{*}{ Grout amount/L } & Simulation & 1839 & 2466 & 3193 & 3110 & 1748 & 1513 & 1554 & 2110 & 1755 & 735 \\
& Measurement & 2674 & 2982 & 2493 & 2509 & 2674 & 2515 & 2451 & 1121 & 2732 & 2513 \\
& Difference \% & -31.2 & -17.3 & 28.0 & 24.0 & -34.6 & -39.8 & -36.6 & 88.2 & -35.8 & -70.8 \\
\hline \multirow{2}{*}{$\begin{array}{l}\text { Injection cement } \\
\text { content/kg }\end{array}$} & Simulation & 2082 & 2831 & 3766 & 3643 & 2003 & 1696 & 1734 & 2438 & 2005 & 627 \\
& Measurement & 2267 & 2592 & 2015 & 2069 & 2263 & 2042 & 1950 & 393 & 2320 & 1787 \\
& Difference \% & -8.1 & 9.2 & 86.9 & 76.1 & -11.5 & -16.9 & -11.1 & 519.7 & -13.6 & -64.9 \\
\hline
\end{tabular}

shown in Figure 5. When the hole spacing was $2.0 \mathrm{~m}$, that is, the distance from the drilling center was $1.0 \mathrm{~m}$, it can meet the design impermeable standard, which is less than $1 \mathrm{Lu}$. When the hole spacing was $2.6 \mathrm{~m}$, it can meet the impermeable standards, which is less than $3 \mathrm{Lu}$.

4.3. Reliability Analysis. The reliability of grouting effect needed to be analyzed due to the randomness of MonteCarlo method. Under the given grouting pressure, respective simulations on multiple sets of different fracture networks were conducted to obtain different calculation results of corresponding number of times. Making a statistical analysis of the distribution of computed results, the reliability of the results can be examined by obtaining the specific values in different confidence intervals. The Monte-Carlo simulation was conducted with 81 times, and the statistical distribution of the calculated results in Stage 9 is shown in Table 3. Corresponding to different confidence intervals, the grout diffusion distances with the permeability rate less than $1 \mathrm{Lu}$ in each grouting stage are shown in Table 4 and Figure 6.

As for the simulation results with $100 \%$ confidence, the grouted stage whose diffusion distance away from the drilling center was the smallest and also satisfied the design standard was Stage $9(45.5-50.5 \mathrm{~m})$, and the hole spacing in this stage was $1.8 \mathrm{~m}$, less than the design value, $2 \mathrm{~m}$. Among the 81 simulation results, only one result, whose water permeability after grouting at a distance of $1 \mathrm{~m}$ from the drilling center was $1.0492 \mathrm{Lu}$, was slightly more than the design standard. Thus, the analysis of simulation results showed that the hole spacing of $2 \mathrm{~m}$ can satisfy the impermeable standard because the confidence was nearly $99 \%$. 


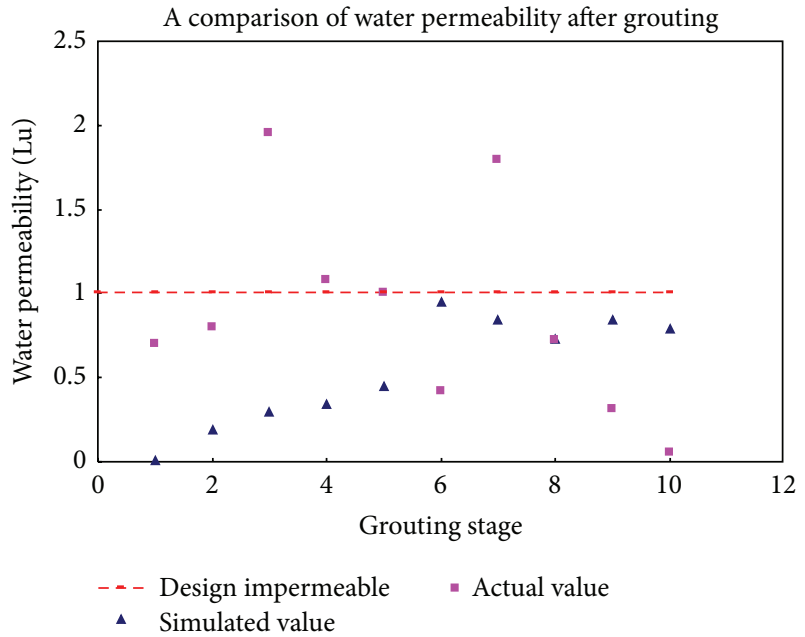

(a)

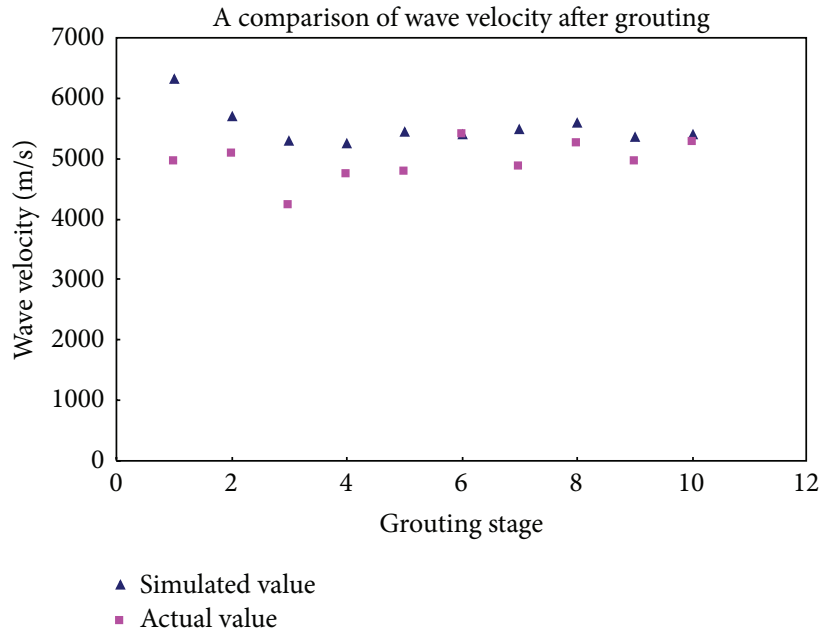

(b)

FIGURE 4: Comparison of the grouting effect after grouting with $1 \mathrm{~m}$ as the design diffusion radius.

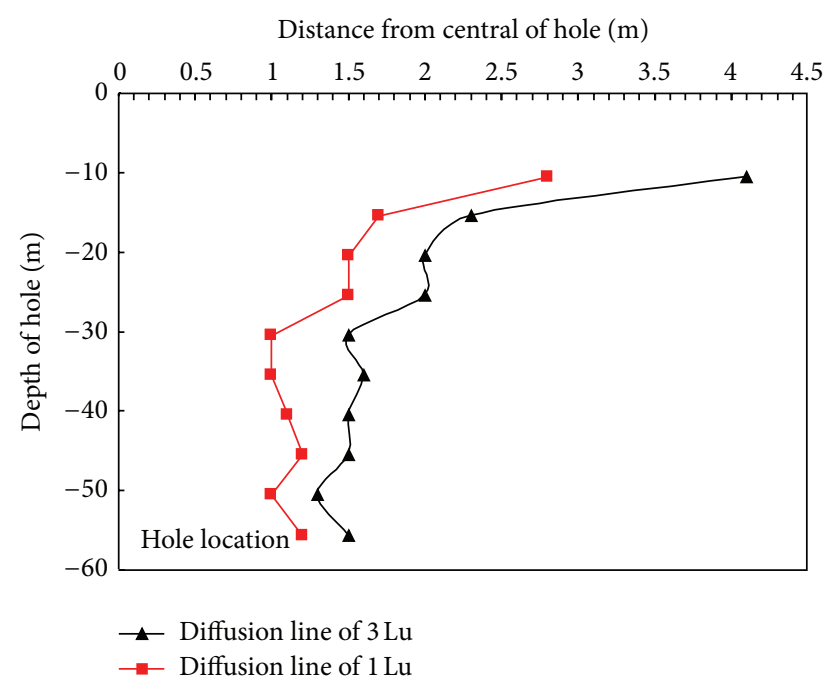

FIGURE 5: Distribution of the water permeability at different distances.

As for the simulation results with $95 \%$ confidence, the grouted stages whose diffusion distance away from the drilling center was the smallest and also satisfied the design standard were Stage $8(40.5-45.5 \mathrm{~m})$ and Stage 9. The hole distance in these two stages was $2.2 \mathrm{~m}$, more than the design value, $2 \mathrm{~m}$. Therefore, if the simulation value with that confidence was adopted, the hole spacing could be optimized to $2.2 \mathrm{~m}$, an increase of $0.2 \mathrm{~m}$ over the design value, directly saving up to $10 \%$ of the project amount.

As for the simulation results with $80 \%$ confidence, the grouted stages whose diffusion distance away from the drilling center was the smallest and also satisfied the design standard were Stage 6 (30.5-35.5 m), Stage 8, and Stage 9. The hole spacing in these three stages was $2.4 \mathrm{~m}$. It should not be applied directly due to its $80 \%$ confidence.

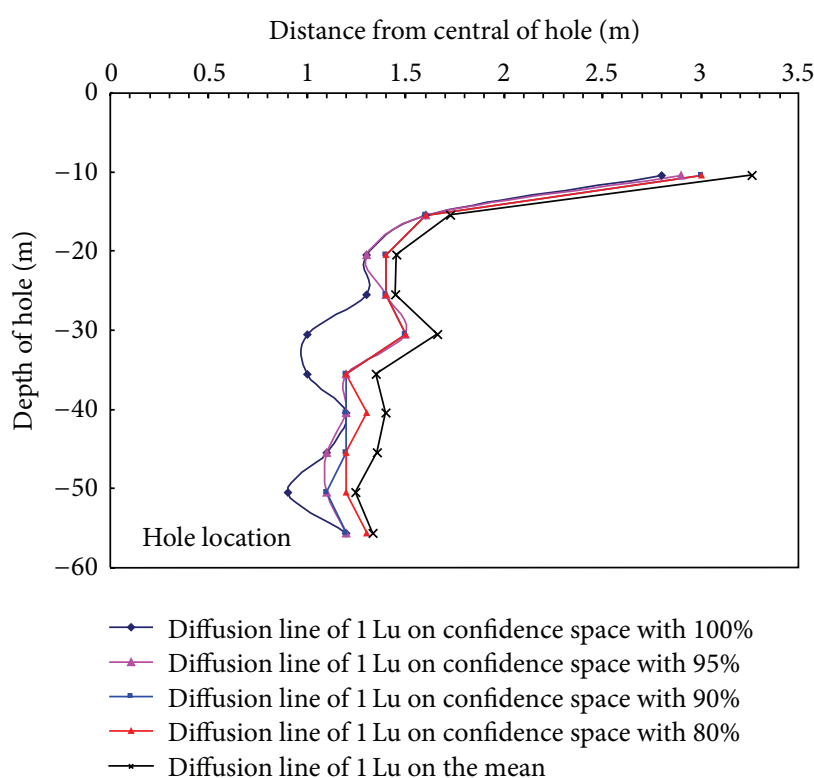

FIGURE 6: Probability of the distance in each grouting stage with permeability rate less than $1 \mathrm{Lu}$.

\section{Conclusion}

Based on the mutual coupling of the diffusion process of cement grouting in the fissures and the deformation of rock mass under grouting pressure, the diffusion and filling process for cement grouting in $\mathrm{V}$-diabase grouting test in the Dagang Mountain Hydropower Station was simulated using the finite element method, considering the time variability of rheological parameters of cement grout, the changes in the fissure opening, and the precipitation law of the cement particles. Distribution of the permeability rate in grouted rock mass was analyzed, and the reliability and optimization 
TABLE 3: Statistical distribution of permeability rate after grouting in Stage 9 at different distances.

\begin{tabular}{|c|c|c|c|c|c|c|}
\hline Distance/m & $\begin{array}{c}\text { Permeability rate of } \\
\text { average/Lu }\end{array}$ & Variance & $\begin{array}{c}\text { Coefficient of } \\
\text { variation }\end{array}$ & Maximum/Lu & Minimum/Lu & $\begin{array}{c}\text { Probability less than } \\
1 \mathrm{Lu} / \%\end{array}$ \\
\hline 0.1 & 0.3044 & 0.0166 & 0.0545 & 0.3428 & 0.2684 & $100 \%$ \\
\hline 0.2 & 0.3029 & 0.0166 & 0.0548 & 0.3419 & 0.2667 & $100 \%$ \\
\hline 0.3 & 0.301 & 0.0166 & 0.0552 & 0.3405 & 0.2647 & $100 \%$ \\
\hline 0.4 & 0.2986 & 0.0166 & 0.0556 & 0.3386 & 0.2623 & $100 \%$ \\
\hline 0.5 & 0.3008 & 0.0215 & 0.0715 & 0.3976 & 0.2596 & $100 \%$ \\
\hline 0.6 & 0.3121 & 0.0284 & 0.0910 & 0.3967 & 0.261 & $100 \%$ \\
\hline 0.7 & 0.3372 & 0.0409 & 0.1213 & 0.4859 & 0.2703 & $100 \%$ \\
\hline 0.8 & 0.3856 & 0.0607 & 0.1574 & 0.716 & 0.2887 & $100 \%$ \\
\hline 0.9 & 0.4626 & 0.0895 & 0.1935 & 0.9654 & 0.3131 & $100 \%$ \\
\hline 1.0 & 0.5848 & 0.1182 & 0.2021 & 1.0492 & 0.3098 & $98.77 \%$ \\
\hline 1.1 & 0.7047 & 0.1459 & 0.2070 & 1.2203 & 0.3818 & $97.53 \%$ \\
\hline 1.2 & 0.8611 & 0.1724 & 0.2002 & 1.428 & 0.448 & $85.19 \%$ \\
\hline 1.3 & 1.1571 & 0.2501 & 0.2161 & 2.1088 & 0.7335 & $32.10 \%$ \\
\hline 1.4 & 1.4857 & 0.315 & 0.2120 & 2.9922 & 0.8091 & $2.47 \%$ \\
\hline 1.5 & 1.8819 & 0.4215 & 0.2241 & 3.506 & 0.8186 & $1.23 \%$ \\
\hline 1.6 & 2.4183 & 0.5183 & 0.2143 & 4.2533 & 1.4244 & $0 \%$ \\
\hline
\end{tabular}

TABLE 4: Probability of distance in each grouting stage with the permeability rate less than $1 \mathrm{Lu}$.

\begin{tabular}{lccccccccccc}
\hline \multirow{2}{*}{ Different confidence from the drilling center/m } \\
& \multicolumn{19}{c}{ Stage 1 } & Stage 2 & Stage 3 & Stage 4 & Stage 5 & Stage 6 & Stage 7 & Stage 8 & Stage 9 & Stage 10 \\
\hline $100 \%$ & 2.8 & 1.6 & 1.3 & 1.3 & 1.0 & 1.0 & 1.2 & 1.1 & 0.9 & 1.2 \\
$95 \%$ & 2.9 & 1.6 & 1.3 & 1.4 & 1.5 & 1.2 & 1.2 & 1.1 & 1.1 & 1.2 \\
$90 \%$ & 3.0 & 1.6 & 1.4 & 1.4 & 1.5 & 1.2 & 1.2 & 1.2 & 1.1 & 1.2 \\
$80 \%$ & 3.0 & 1.6 & 1.4 & 1.4 & 1.5 & 1.2 & 1.3 & 1.2 & 1.2 & 1.3 \\
Mean & 3.261 & 1.728 & 1.453 & 1.450 & 1.660 & 1.351 & 1.402 & 1.357 & 1.247 & 1.337 \\
\hline
\end{tabular}

of the hole spacing design were discussed. The following conclusions were derived according to the analysis.

(1) Through numerical simulation of the grout diffusion, the permeability rate of the grouted rock mass was obtained, and the simulation results were compared with the practical grouting results. This showed that the errors in the grouting process, including the injection rate, the accumulated grouting volume, and the accumulated injection cement content, were acceptable. The simulation process basically reflects the grouting process. The simulated grouting results, the water permeability, and the acoustic wave velocity after grouting were comparable with the practical grouting results.

(2) Simulation results of the grouting process with the same parameters for many times showed that various quantities had good repeatability, such as grouting time, the accumulated grouting volume, the accumulated injection cement content, the permeability rate, and the acoustic wave velocity after grouting. Most of the variation coefficients were less than 0.3 and distributed uniformly. The simulating results had better reliability in general.
(3) The numerical simulation results showed that the grouting pressure, the hole spacing, and the materials used in the test area were essentially reasonable and practicable. Diffusion distance of the grout satisfying the design permeability standard could reach $1 \mathrm{~m}$ with the confidence of approximately $100 \%$. Thus, the hole spacing of $2 \mathrm{~m}$ was reliable.

(4) The analysis results showed that, when the confidence approached 95\%, diffusion distance of the grout, which satisfied the design permeability standard, could reach $1.1 \mathrm{~m}$; that is, the hole spacing reached $2.2 \mathrm{~m}$. This demonstrated a probability of optimization along with a finer optimization precision when compared with the accuracy of $0.5 \mathrm{~m}$ that is commonly used.

(5) There were differences between the numerical simulating process curve and the measurements. The grouting time, the rising curve of grouting pressure, and the assumptions adopted in the simulation might be the main reason for the differences. It was indicated that the simulation results tended to be more perfect with fewer restrictions. 


\section{Conflict of Interests}

The authors declare that there is no conflict of interests regarding the publication of this paper.

\section{Acknowledgment}

This research is supported by the National Natural Science Fund Project no. 51279217.

\section{References}

[1] S. Maghous, Z. Saada, L. Dormieux, J. Canou, and J. C. Dupla, "A model for in situ grouting with account for particle filtration," Computers and Geotechnics, vol. 34, no. 3, pp. 164-174, 2007.

[2] A. Contini, A. Cividini, and G. Gioda, "Numerical evaluation of the surface displacements due to soil grouting and to tunnel excavation," International Journal of Geomechanics, vol. 7, no. 3, pp. 217-226, 2007.

[3] H. F. Schweiger, C. Kummerer, R. Otterbein, and E. Falk, "Numerical modelling of settlement compensation by means of fracture grouting," Soils and Foundations, vol. 44, no. 1, pp. 7186, 2004.

[4] G. Modoni, P. Croce, and L. Mongiovì, "Theoretical modelling of jet grouting," Geotechnique, vol. 56, no. 5, pp. 335-347, 2006.

[5] E. D. Lees and A. Naudts, "New developments in rock and soil grouting design and evaluation," Dam Engineering, vol. 6, no. 1, pp. 111-129.

[6] C. Wisser, C. E. Augarde, and H. J. Burd, "Numerical modelling of compensation grouting above shallow tunnels," International Journal for Numerical and Analytical Methods in Geomechanics, vol. 29, no. 5, pp. 443-471, 2005.

[7] Y. Xiaodong, Analysis of Surface Uplift Grouting in Rock Foundation, China Institute of Water Resources and Hydropower Research, Beijing, China, 1987, Chinese.

[8] L. Hassler et al., "Simulation of grouting in jointed rock," in Proceedings of the 6th International Conference on Rock Mechanics, 1987.

[9] L. Hässler, U. Håkansson, and H. Stille, "Computer-simulated flow of grouts in jointed rock," Tunnelling and Underground Space Technology incorporating Trenchless, vol. 7, no. 4, pp. 441446, 1992.

[10] Z. Hao, A Research on the Rock Grouting Behavior and Its Computer Simulation, Doctoral Dissertation of Northeastern University, Shenyang, China, 1998, Chinese.

[11] M. Eriksson, H. Stille, and J. Andersson, "Numerical calculations for prediction of grout spread with account for filtration and varying aperture," Tunnelling and Underground Space Technology, vol. 15, no. 4, pp. 353-364, 2000.

[12] M. Young, M. X. Chen, and Y. N. HE, "Simulating experiment for grouting seepage in rockmass," Journal of Experimental Mechanics, vol. 16, no. 1, pp. 105-112, 2001.

[13] P.-P. Luo, Y.-M. Zhu, Y.-M. Zhao, and S. He, "Numerical simulation of grouting in rock mass," Chinese Journal of Geotechnical Engineering, vol. 27, no. 8, pp. 918-921, 2005.

[14] P. Fu and X. D. Yang, "Study on the effect assessment and the numerical simulation of the cement in fractured rockmass," in Proceedings of the 11th Symposium of the National Water Resources and Hydropower ground and foundation engineering technology, pp. 9-17, Water Power Press, Beijing, China, 2011.
[15] Shannon \& Wilson, Geotechnical Consultants, Cement Grout Flow Behavior in Fractured Rock, 1987.

[16] M. Eriksson, M. Friedrich, and C. Vorschulze, "Variations in the rheology and penetrability of cement-based grouts-an experimental study," Cement and Concrete Research, vol. 34, no. 7, pp. 1111-1119, 2004.

[17] J. Mirza, M. S. Mirza, V. Roy, and K. Saleh, "Basic rheological and mechanical properties of high-volume fly ash grouts," Construction and Building Materials, vol. 16, no. 6, pp. 353-363, 2002.

[18] G. Rudolph, S. luo et al., Studies of the Setting Behavior of Cement Suspensions, Institute of Nuclear waste disposal engineering, 1982.

[19] R. Gothäll and H. Stille, "Fracture dilation during grouting," Tunnelling and Underground Space Technology, vol. 24, no. 2, pp. 126-135, 2009.

[20] C. E. Renshaw, "On the relationship between mechanical and hydraulic apertures in rough-walled fractures," Journal of Geophysical Research, vol. 100, no. 12, pp. 24-636, 1995.

[21] W.-G. Qiao, Y.-X. Zhang, X.-H. Song, and T. Zhao, "Flow deposition mechanism of cement slurry in rock ass fractures," Rock and Soil Mechanics, vol. 25, pp. 14-16, 2004 (Chinese).

[22] S. C. Gu, P. L. Su et al., Numerical Simulation of the Fracture Tip Under the Grouting Pressure, Ground Treatment, Chinese.

[23] S. G. Du, The Engineering Properties of the Rockmass on the Structure Surface, Earthquake Press, Beijing, China, 1999, Chinese.

[24] G. Louis, A Study of Ground Water Flow in Jointed Rock and its Influence on the Stability of Rock Masses, Rock Mechanics Research Report, London, UK, 1969. 


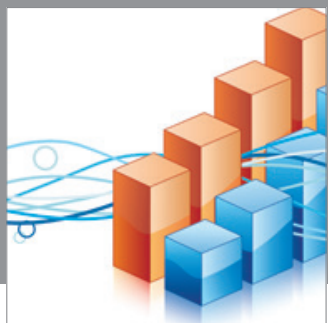

Advances in

Operations Research

mansans

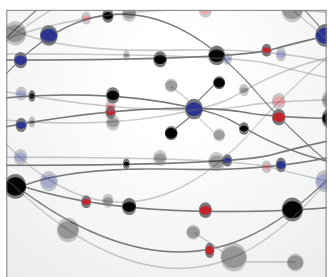

The Scientific World Journal
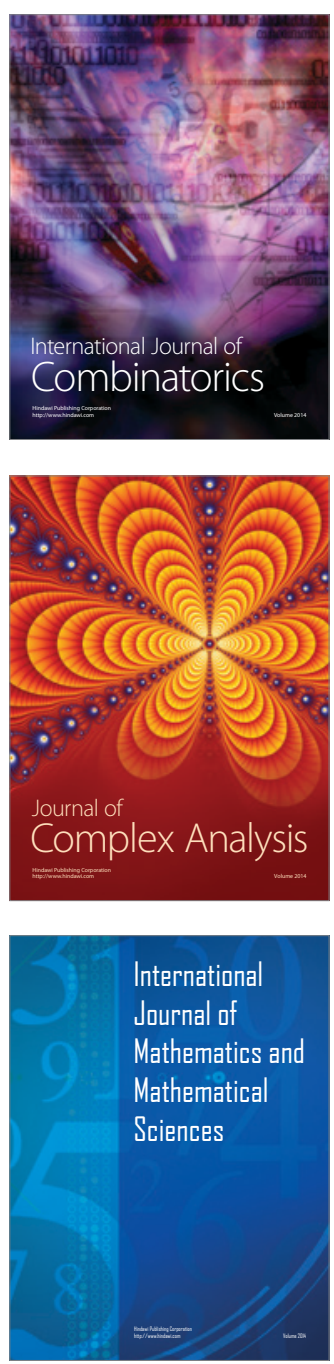
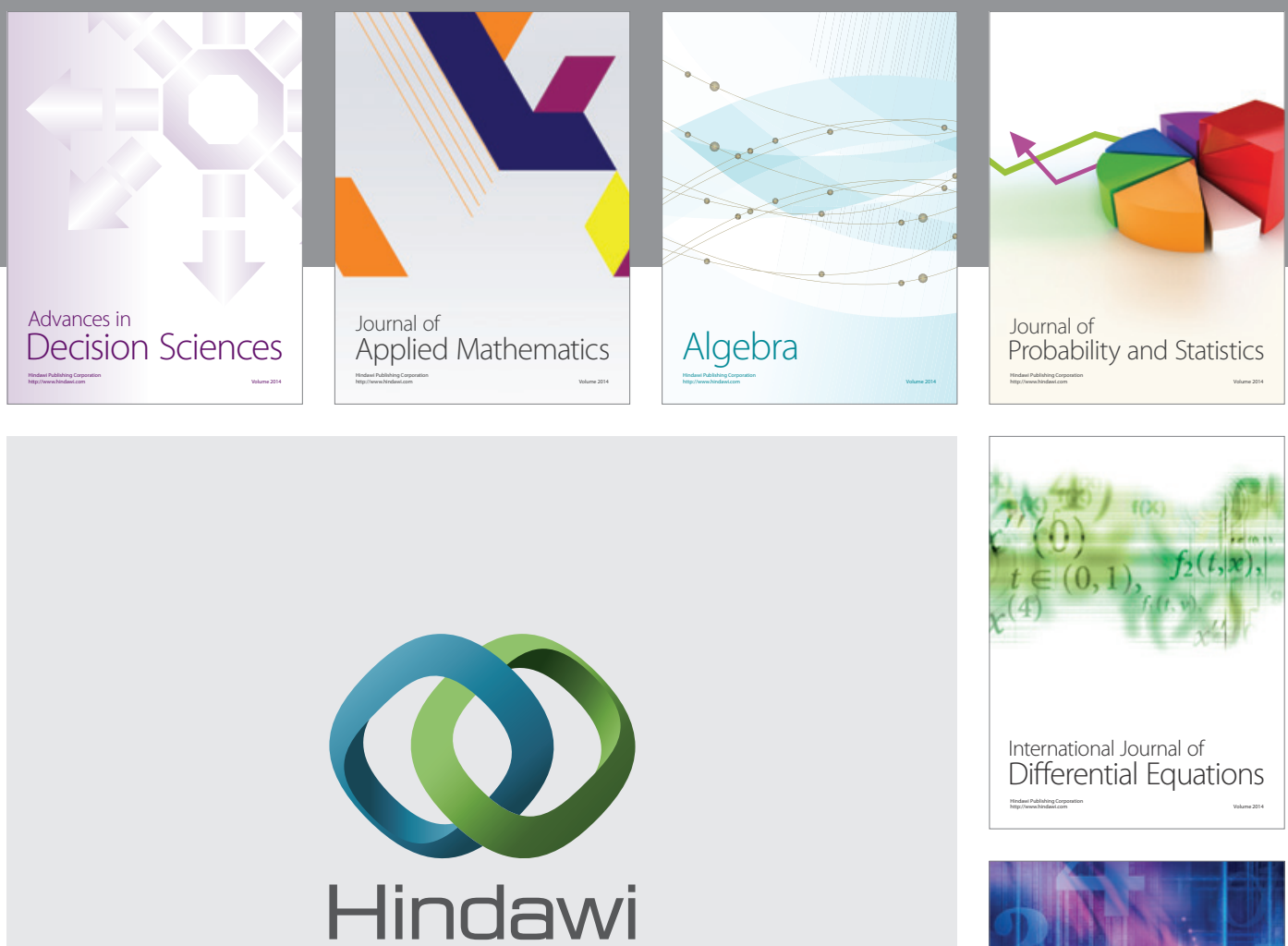

Submit your manuscripts at http://www.hindawi.com
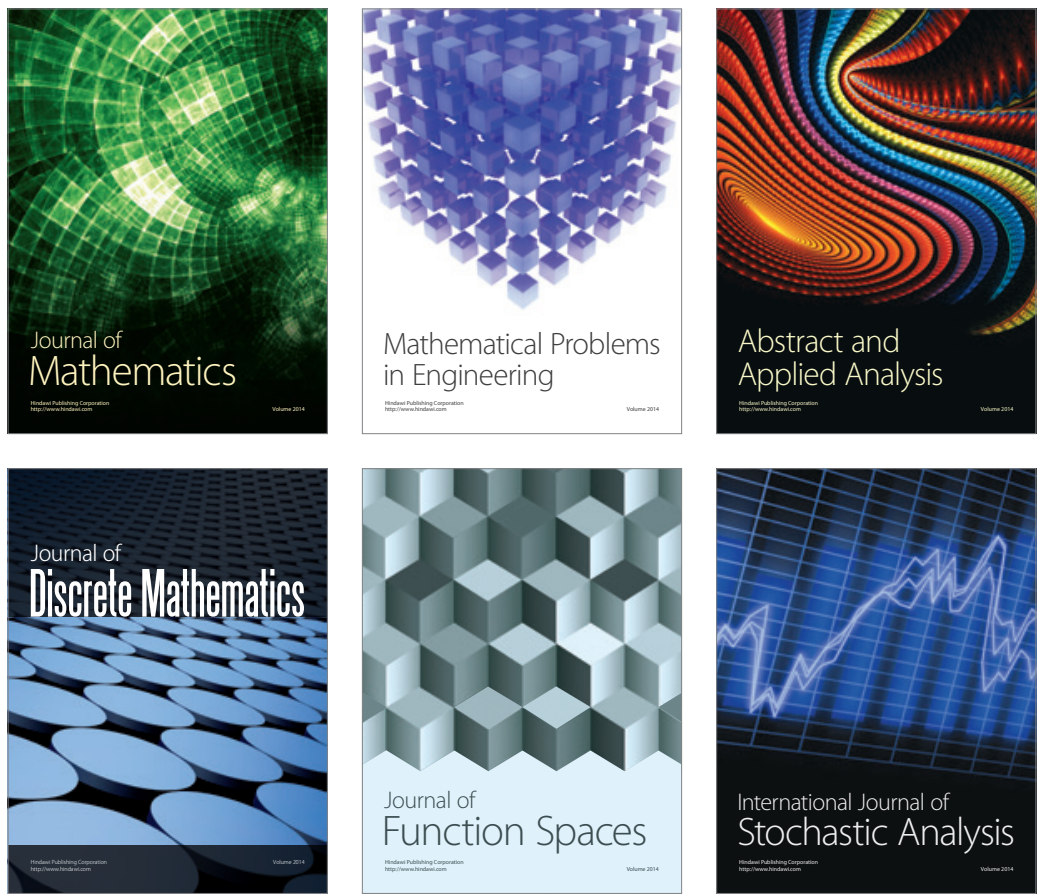

Journal of

Function Spaces

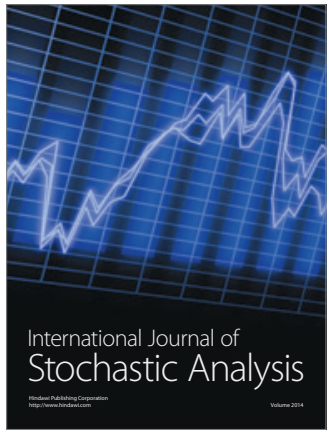

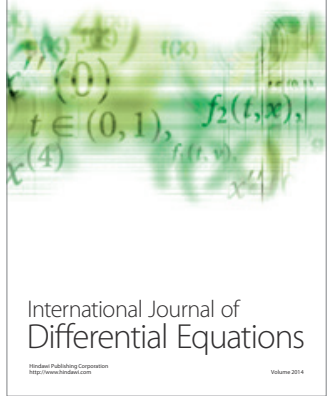
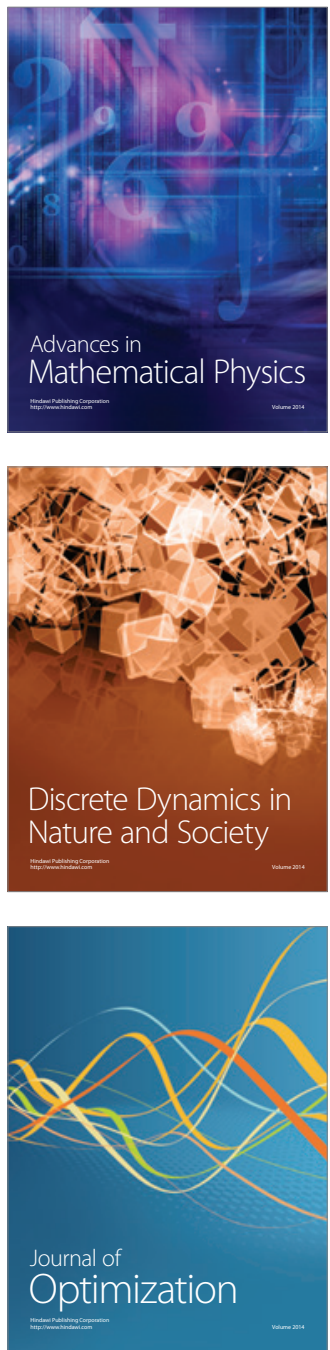